\title{
Genomic and transcriptional heterogeneity-based precision in personalized treatment for breast cancer
}

\author{
Persefoni Antoniou ${ }^{1,2}$, Demosthenes E Ziogas ${ }^{1,3}$, Aikaterini Vlachioti1,4, Eustathios G \\ Lykoudis $^{5}$, Michail Mitsis ${ }^{2,5}$ \& Dimitrios H Roukos*,1,2,5,6 \\ ${ }^{1}$ Centre for Biosystems \& Genome Network Medicine, loannina University, loannina, Greece \\ ${ }^{2}$ Department of Surgery, loannina University Hospital, loannina, Greece \\ ${ }^{3}$ Department of Surgery, 'G Hatzikosta' General Hospital, loannina, Greece \\ ${ }^{4}$ Department of Gynecology, General Hospital, Preveza, Greece \\ ${ }^{5}$ Cancer Biobank Centre, Department of Medicine, University of Ioannina, Ioannina, Greece \\ ${ }^{6}$ Department of Systems Biology, Biomedical Research Foundation of the Academy of Athens (BRFAA), Athens, Greece \\ *Author for correspondence: Tel.: +30 265100 5572; droukos@uoi.gr
"However, acquired resistance, late relapse and cancer-related death rates remain major challenges."

First draft submitted: 25 March 2019; Accepted for publication: 8 April 2019; Published online: 11 September 2019

Keywords: biomarkers $\bullet$ breast cancer $\bullet$ genome $\bullet$ oncotargets $\bullet$ precision oncology $\bullet$ transcriptome

The greatest success in the clinical standardization of personalized medicine among all cancer types has been noted in breast cancer [1,2]. However, acquired resistance, late relapse and cancer-related death rates remain major challenges. In this editorial, we discuss the potential to overcome these unmet needs with genome and transcriptome-based biomarkers and drugs [3].

In contrast to most other major cancer types, progress is impressive for breast cancer $[1,2]$. Combining clinicopathologic characteristics, tumour, node, metastasis (TNM) staging and molecular classification for personalized decision-making treatment, exciting survival rates have recently been reported in early, advanced resectable and metastatic disease [2,4]. Particularly, the dual combination of pertuzumab or neratinib added to standard trastuzumab plus chemotherapy for HER2-positive breast cancer has dramatically improved oncological outcomes of patients in the adjuvant [4] and metastatic setting [5].

Despite these advances for women with HER2-positive subtype, a proportion of these women face a risk of acquired resistance and late relapse [3]. Women with triple-negative breast cancer face the risk of both early and late relapse, considering that chemotherapy alone is available while no targeted drugs have been approved for these patients [6].

One of the most exciting and rational research strategies to overcome the current unmet research and clinical needs is the emerging genome and transcriptome analysis in time and space [7]. Technological progress with the validity of next-generation sequencing (NGS) opens new predictive and therapeutic horizons for further improvement of oncological outcomes. We summarize these advances to shift from conventional personalized medicine to the emerging precise prognostic and drug prediction-based individualized management of breast cancer.

\section{Personalized management of breast cancer}

Breast cancer represents the most successful paradigm, among all cancer types, of personalized strategy in both prevention and treatment settings. Major discoveries in single mutated and amplified genes over the past two decades have been rapidly translated into clinical practice, providing impressive survival benefits for millions of women. 


\section{Personalized prevention for hereditary breast-ovarian cancer syndrome}

Individualized decision making for preventive intervention is based on women with significant family history of breast and/or ovarian cancer, clinical, pathological, molecular and genetic criteria. For example, breast cancer aggressiveness differs among $B R C A 1$ and $B R C A 2$ mutation carriers. Women with $B R C A 1$ mutation have a risk of $75 \%$ to develop breast cancer and over $60 \%$ of ovarian cancer while these figures for $B R C A 2$ women are 75 and $20 \%$, respectively [8]. Moreover, the predisposition for the development of poor prognosis in triple negative and good prognosis in estrogen receptor positive $(\mathrm{ER}+)$ /progesterone receptor positive $(\mathrm{PR}+)$ subtypes are 60-80\% for $B R C A 1$ and $B R C A 2$, respectively. In addition, prophylactic bilateral mastectomy and/or bilateral salpingooophorectomy has been the standard option for women with $B R C A 1 / 2$ germline mutation, considering all the above-mentioned characteristics with the age of risk prevention surgery to be 5-10 years earlier than disease diagnosis in the younger relative.

\section{Treatment advances}

Established multimodal treatment including surgery, radiotherapy and chemotherapy as well as targeted therapy has substantially improved therapeutic resistance, relapse and cancer-related death rates [1,2]. Decision making is currently guided by the results from randomized control trials (RCTs). Clinico-pathologic and molecular characteristics, as well as TNM staging guide personalized treatment. Multiple drugs have been developed and approved by the FDA including beyond cytotoxic agents, anti-estrogens and aromatase inhibitors for ER+/PR+ women, as well as anti-HER2 drugs. The most impressive progress in targeted therapy among all cancer types has recently been noted in dual blockade of HER2 signaling pathway, adding the monoclonal antibody pertuzumab or the small tyrocine kinase inhibitor (pTKI) to standard systemic therapy of trastuzumab plus chemotherapy. This combined targeted therapy has achieved 3-year disease-free survival over 90\% [9]. Moreover, substantial progress has been observed in the treatment of a subgroup of patients with hormone receptor positive (HR), HER2 negative metastatic postmenopausal women with the addition of palpociclib and ribociclib. Finally, even for triple-negative disease with appropriate chemotherapeutic regimen in an absence of targeted drugs, 5-year disease-free survival of $77 \%$ has recently been reported [6].

Although important research advances have been translated into improvement of oncological outcomes for breast cancer, a significant proportion of women continue to face a high risk of treatment failure.

\section{Genomic studies for breast cancer}

The evidence of extensive genetic heterogeneity in cancer including breast tumors has revolutionized biomedical research [10]. Over the past 10 years, an explosion in NGS studies has already substantially improved basic and translational research for breast cancer. Static and spatiotemporal genome analysis with NGS including based on single biopsy and multiple samples respectively have already reported exciting basic and translational research discoveries with potential clinical implications.

Single tumoral biopsy has already provided wide clinical implications with targeted-NGS (tNGS) for a panel of known genes. Furthermore, whole exome (WES), whole genome sequencing (WGS) analyses with or without RNA-seq, following recent recommendation on large-scale studies [10], have already reported the identification of many breast cancer driver genes, mutations and breast-cancer specific oncotargets [10]. However this static genome analysis is associated with strong limitations to overcome drug resistance considering intra-tumor genetic heterogeneity and dynamic evolution of genomic clones.

\section{Spatiotemporal emergence of genetic \& genomic heterogeneity}

Genome analysis in time and space, considering emerging data on intratumor heterogeneity (ITH) and dynamic evolution of genomic clones according to Darwinian principles [11] has been developed to overcome therapeutic resistance and relapse in individual patients. Yates et al. analyzed 303 samples from 50 breast cancer patients applying WGS and tNGS in 29 and 290 samples, respectively, identifying ITH and druggable mutations in 13 of the 50 patients [12]. Furthermore, they used multi-regional tNGS before and after NAT, detecting new subclones in 5 of 18 patients after NAT that did not exist at the pre-NAT samples [12]. Multiple studies on patients with metastatic disease, applying serial circulating-free DNA-NGS (cfDNA-NGS), have shown that cfDNA-NGS can be used as a predictive and monitoring tool, detecting recurrence even before clinical or imaging relapse [13-16]. 


\section{Unmet needs}

Despite this promising data on genomic and transcriptomic landscape, multiple challenges have emerged. Most of the studies have not considered patients' linic-pathologic characteristics, imaging results and TNM staging. In addition, these studies are limited by a small number of patients and samples, in an absence of prospective protocol. Therefore, the principle of evidence-based medicine, required for clinical implications, have not been met.

\section{Future perspective \& conclusion}

Although there has been a rapid progress in the treatment of HER2-positive disease, with the recent dual HER2targeted therapy with pertuzumab plus trastuzumab, acquired resistance and relapse remain unresolved problems. For the triple-negative subgroup of patients, chemotherapy continues to remain the only standard treatment without any approved targeted drugs, as reflected by intrinsic and acquired drug resistance and associated with high early and late recurrence rates [1]. The genomic and transcriptomic exploration provides exciting perspectives for overcoming the mentioned unmet needs, but the available data are yet inconclusive.

Emerging solutions are rapidly developed into major research directions. First, the evaluation of spatiotemporal development of genomic clones responsible for drug resistance within a new generation of clinic-genomic trials [17]. Second, to identify cell by cell genetic, genomic and transcriptional heterogeneity [18] applying a breakthrough combination of single-cell transcriptomics and genome editing tools [19].

Indeed, shifting from conventional standard clinical trials to a new generation of spatiotemporal patient-centric trials is necessary for the potential establishment of ITH and matched serial cfDNA-NGS as prognostic and predictive robust biomarkers. This comprehensive intra-individual landscape considering both tumoral and liquid biopsies analysis represents an exciting tool to optimize personalized treatment of breast cancer following the rules of high quality clinical medicine. In a more distant future, on the basis of ENCODE project of healthy human genome, rises the potential for the development of next-generation drugs disrupting the aberrant transcriptional networks in cancer [20-22].

In summary, the combination of clinico-pathologic and genetic characteristics has achieved a substantial progress toward individualization of breast cancer therapy. The challenges of early and late acquired resistance and relapse could be overcome with an appropriate design and conduction of new generation of spatiotemporal genomic trials in a medium-term perspective. However, the optimization of personalized therapy for women with breast cancer will require the valid identification of comprehensive intraindividual coding and noncoding mutational landscape with both bulk NGS and single-cell RNA sequencing and a combination of single-cell RNA sequencing with CRISPR-CAS9 systems.

\section{Financial \& competing interests disclosure}

The authors have no relevant affiliations or financial involvement with any organization or entity with a financial interest in or financial conflict with the subject matter or materials discussed in the manuscript. This includes employment, consultancies, honoraria, stock ownership or options, expert testimony, grants or patents received or pending, or royalties.

No writing assistance was utilized in the production of this manuscript.

\section{References}

1. Network NCC. National Comprehensive Cancer Network (2019). www.nccn.org/

2. Siegel RL, Miller KD, Jemal A. Cancer statistics, 2019. CA 69(1), 7-34 (2019).

3. Kyrochristos ID, Ziogas DE, Roukos DH. Dynamic genome and transcriptional network-based biomarkers and drugs: precision in breast cancer therapy. Medicinal Res. Rev. 39(3), 1205-1227 (2018).

4. Martin M, Holmes FA, Ejlertsen B et al. Neratinib after trastuzumab-based adjuvant therapy in HER2-positive breast cancer (ExteNET): 5-year analysis of a randomised, double-blind, placebo-controlled, Phase III trial. Lancet Oncol. 18(12), 1688-1700 (2017).

5. Swain SM, Kim SB, Cortes J et al. Pertuzumab, trastuzumab, and docetaxel for HER2-positive metastatic breast cancer (CLEOPATRA study): overall survival results from a randomised, double-blind, placebo-controlled, Phase 3 study. Lancet Oncol. 14(6), 461-471 (2013).

6. Bell R, Brown J, Parmar M et al. Final efficacy and updated safety results of the randomized Phase III BEATRICE trial evaluating adjuvant bevacizumab-containing therapy in triple-negative early breast cancer. Ann. Oncol. 28(4), 754-760 (2017).

7. Roukos DH. Spatiotemporal diversification of intrapatient genomic clones and early drug development concepts realize the roadmap of precision cancer medicine. Drug Discov. Today 22(8), 1148-1164 (2017).

8. Lerner-Ellis J, Khalouei S, Sopik V, Narod SA. Genetic risk assessment and prevention: the role of genetic testing panels in breast cancer. Exp. Rev. Anticancer Ther. 15(11), 1315-1326 (2015). 
9. Von Minckwitz G, Procter M, De Azambuja E et al. Adjuvant pertuzumab and trastuzumab in early HER2-positive breast cancer. $N$. England J. Med. 377(2), 122-131 (2017).

10. Lawrence MS, Stojanov P, Mermel $\mathrm{CH}$ et al. Discovery and saturation analysis of cancer genes across 21 tumour types. Nature 505(7484), 495-501 (2014).

11. Eirew P, Steif A, Khattra J et al. Dynamics of genomic clones in breast cancer patient xenografts at single-cell resolution. Nature 518(7539), 422-426 (2015).

12. Yates LR, Gerstung M, Knappskog $S$ et al. Subclonal diversification of primary breast cancer revealed by multiregion sequencing. Nat. Med. 21(7), 751-759 (2015).

13. Dawson SJ, Tsui DW, Murtaza M et al. Analysis of circulating tumor DNA to monitor metastatic breast cancer. N. England J. Med. 368(13), 1199-1209 (2013).

14. Olsson E, Winter C, George A et al. Serial monitoring of circulating tumor DNA in patients with primary breast cancer for detection of occult metastatic disease. EMBO Mol. Med. 7(8), 1034-1047 (2015).

15. Murtaza M, Dawson SJ, Tsui DW et al. Non-invasive analysis of acquired resistance to cancer therapy by sequencing of plasma DNA. Nature 497(7447), 108-112 (2013).

16. Ma F, Zhu W, Guan Y et al. ctDNA dynamics: a novel indicator to track resistance in metastatic breast cancer treated with anti-HER2 therapy. Oncotarget 7(40), 66020-66031 (2016).

17. Ziogas DE, Kyrochristos ID, Roukos DH. Discovering novel valid biomarkers and drugs in patient-centric genomic trials: the new epoch of precision surgical oncology. Drug Discov. Today 23(11), 1848-1872 (2018).

18. Ben-David U, Siranosian B, Ha G et al. Genetic and transcriptional evolution alters cancer cell line drug response. Nature 560(7718), 325-330 (2018).

19. Kyrochristos I, Ziogas D, Roukos D. Drug resistance: origins, evolution and characterization of genomic clones and tumor ecosystem to optimize precise individualized therapy. Drug Discov. Today 24(6), 1281-1294 (2019).

20. Roukos DH. Crossroad between linear and nonlinear transcription concepts in the discovery of next-generation sequencing systems-based anticancer therapies. Drug Discov. Today 21(4), 663-673 (2016).

21. Lesterhuis WJ, Bosco A, Millward MJ, Small M, Nowak AK, Lake RA. Dynamic versus static biomarkers in cancer immune checkpoint blockade: unravelling complexity. Nat. Rev. Drug Discov. 16(4), 264-272 (2017).

22. Fellmann C, Gowen BG, Lin PC, Doudna JA, Corn JE. Cornerstones of CRISPR-Cas in drug discovery and therapy. Nat. Rev. Drug Discov. 16(2), 89-100 (2017). 